\title{
A Theoretical Study of Vibrational Properties of Neutral and Cationic $\mathrm{B}_{12}$ Clusters
}

\author{
KAH CHUN LAU, MRINALINI DESHPANDE, RAVINDRA PANDEY \\ Department of Physics, Michigan Technological University, Houghton, MI 49931
}

Received 28 July 2004; accepted 10 November 2004

Published online 18 January 2005 in Wiley InterScience (www.interscience.wiley.com).

DOI 10.1002/qua.20464

\begin{abstract}
Calculations based on density functional theory predict the ground state of $\mathrm{B}_{12}$ and $\mathrm{B}_{12}^{+}$to be a convex planar configuration with $C_{1}$ symmetry. A small ionization-induced structural change in $B_{12}$ is also predicted. It is suggested that multicentered bonds together with delocalized charge density are dominant factors in stabilizing the planar configuration over $3 \mathrm{D}$ configuration for $\mathrm{B}_{12}$ and $\mathrm{B}_{12}^{+}$. The calculated vibrational frequencies lie in the range of $200-1328 \mathrm{~cm}^{-1}$ in which the highfrequency modes are associated with asymmetric stretching of the boron atoms located at the cluster surface. The infrared spectra of both neutral and cationic $B_{12}$ show similar infrared active modes in the high-frequency region, but different modes in the lowfrequency region due to dissimilar atomic charges in the cationic $B_{12}$. The combined effect of charge delocalization and reduced dimensionality is reflected in the calculated static dipole polarizability of these clusters. (C) 2005 Wiley Periodicals, Inc. Int J Quantum Chem 102: 656-664, 2005
\end{abstract}

Key words: clusters; boron; density functional theory; IR spectra

\section{Introduction}

$\mathbf{T}$ he boron compounds show a wide variety of structures and compositions that include single atom, connected pairs, multiple chains, planar networks, and polyhedral groupings. This variety of structures is a consequence of the peculiar elec-

Correspondence to: R. Pandey; e-mail: pandey@mtu.edu

M. Deshpande's permanent address: Department of Physics, H. P. T. Arts and R. Y. K. Science College, Nasik, India. tronic structure of boron, which is characterized by an unpaired electron in the (He) $2 s^{2} 2 p^{1}$ configuration. It can then lead to various hybridization like $\mathrm{sp}, \mathrm{s}^{2} \mathrm{p}$, $\mathrm{sp}^{2}$, and $\mathrm{sp}^{3}$ between boron atoms or by the interactions with other atoms [1]. Although boron chemistry is mainly based on the $\mathrm{B}_{12}$ icosahedra and $\mathrm{B}_{6}$ octahedron units [2-8], recent studies have uncovered 2D structures for small boron clusters. For example, several studies on $\mathrm{B}_{12}^{+}$and $\mathrm{B}_{13}^{+}$have reported the stable planar and quasiplanar structures [9-15] which were found to be different from the conventional 3D cages obtained in boranes $[5,6]$. 
The primary focus of most of the previous theoretical studies on boron clusters [11-24] has been calculations of the structural and electronic properties in which calculations of the vibrational properties of the boron clusters were not performed. Specifically, various isomeric configurations of $B_{12}$ were considered for electronic structure calculations $[11,18,19,23]$, but analysis of the vibrational stability of any of the isomeric configurations was not performed yet. It is well known that calculations of the vibrational properties together with the IR spectra help experimentalists in identifying the cluster configurations.

In this work, we present the results of a theoretical study based on density functional theory (DFT) with a focus on calculations of the vibrational properties of the neutral and cationic $\mathrm{B}_{12}$. Furthermore, we have analyzed molecular orbitals of several conformers of $\mathrm{B}_{12}$ and $\mathrm{B}_{12}^{+}$to establish a general pattern of physics and chemistry of boron clusters. It is expected that such an analysis will reveal an interplay between the the degree of localization of molecular orbitals and the coordination number for the cluster atoms in predicting the stability of configurational isomers of $\mathrm{B}_{12}$. It has recently been pointed out that the ground-state configuration of the neutral and ionized state of a given cluster may not be the same [25]. We will therefore investigate the ionization-induced structural changes in $B_{12}$. We will also calculate, for the first time, the static dipole polarizability of several isomeric configurations of $\mathrm{B}_{12}$ and $\mathrm{B}_{12}^{+}$to assess the variation in the polarizability with the structural arrangement of boron atoms. The presentation of this article is organized as follows: In Section 2, we briefly describe computational details. Results and discussion of the geometrical features, cluster stability, vibrational stability, static dipole polarizability, and a comparison of some of the results obtained using the basis sets $6-31 \mathrm{G}(d, p)$ and $6-311 \mathrm{G}(d f)$ for $\mathrm{B}_{12}$ and $\mathrm{B}_{12}^{+}$clusters, are presented in Section 3. In Section 4, conclusions are given.

\section{Computational Method}

The electronic structure calculations were performed for several neutral and cationic structural isomers of $B_{12}$ using the Gaussian 98 code [26]. All calculations were carried out by solving the KohnSham equations in the DFT framework. We have employed the generalized gradient approximations (GGA) using the functionals of Becke's 3-parameter hybrid exchange functional [27] and the Lee-YangParr correlation functional (B3LYP) and a doublezeta basis set $(6-31 G(d, p))$ in these calculations. Some of the isomers were selected for calculations using the 6-311G $(d f)$ basis set to test the reliability and accuracy of the results obtained using the $6-31 G(d, p)$ basis set. All the structures in this work have been fully optimized by employing the gradient and updated Hessian. The convergence criteria for the gradient and energy were set to $10^{-4}$ hartree/ $\AA$ and $10^{-9}$ hartree respectively. Calculations of the static dipole polarizability was performed by applying an external electric field of strength 0.001 a.u. along the $x, y$, and $z$ axes separately. The stability of the isomeric configurations considered here was assessed by computing the vibrational frequencies under the harmonic approximation with analytical force constants.

\section{Results and Discussion}

\section{STRUCTURES AND ENERGETICS}

Several isomers of $\mathrm{B}_{12}$ considered for DFT calculations in this work were taken from previous studies $[11,18,19,22]$. They can be categorized into planar (2D) and 3D configurations. Figure 1 shows the optimized geometries of some of the low-energy isomers. The details of other configurations considered here can be obtained from one of the authors. The calculated results find the neutral isomers to be in the singlet spin state. Total energy of the 3D structures are always higher than that of the planar structures. The most stable configuration is a convex structure with $C_{1}$ symmetry (i.e., $B_{12}-\mathrm{I}$ ), which is in contrast to the reported $C_{3 v}$ structure as the most stable configuration of $B_{12}[18,19,23]$. The $C_{1}$ configuration (i.e., $B_{12}-I$ ) consists of three $B_{7}$ units which are reported [24] to be the most stable structure of $\mathrm{B}_{7}$. The next low-energy isomer is the $C_{2}$ planar structure (i.e., $\mathrm{B}_{12}-\mathrm{II}$ ), while calculations at the CI/3-21G level of theory [19] find the $D_{2 h}$ structure to be the second-lowest energy isomer for $B_{12}$. Because the CI/3-21G study [19] did not assess the cluster stability by the frequency calculations, both $C_{3 v}$ and $D_{2 h}$ configurations of $B_{12}$ may be associated with imaginary frequencies.

The next configuration in terms of total energy is a cage-type $C_{2 h}$ structure (i.e., $\mathrm{B}_{12}$-III). It is different from the conventional $B_{12}$ icosahedra and can be viewed as four $B_{7}$ units overlapping in the opposite site where each opposite face appears as the lowest 


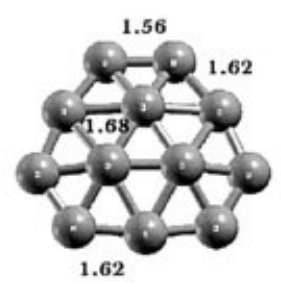

$\mathrm{B}_{12}$-I, singlet

$\mathrm{C}_{1}$

$\mathrm{BE}=4.81 \mathrm{eV} /$ atom

$\Delta E=0.00$

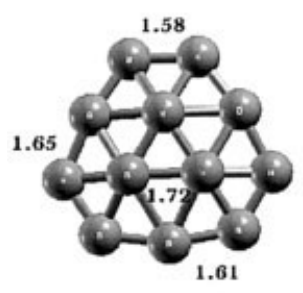

$$
\begin{aligned}
& \mathrm{B}_{12}^{+}-\mathrm{I} \text {, doublet } \\
& \mathrm{C}_{1} \\
& \mathrm{BE}=4.11 \mathrm{eV} / \text { atom } \\
& \Delta E=0.00
\end{aligned}
$$

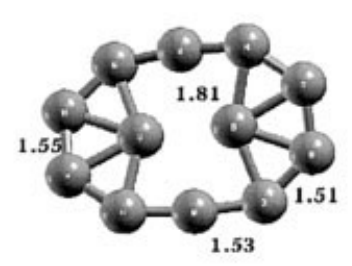

$$
\begin{aligned}
& \mathrm{B}_{12}-\mathrm{II} \text {, singlet } \\
& \mathrm{C}_{2} \\
& \mathrm{BE}=4.68 \mathrm{eV} / \text { atom } \\
& \Delta E=1.56
\end{aligned}
$$

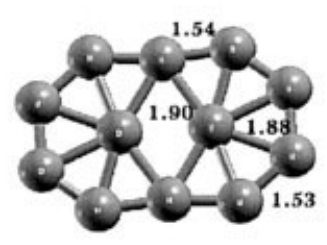

$$
\begin{aligned}
& \mathrm{B}_{12}^{+}-\mathrm{II} \text {, doublet } \\
& \mathrm{C}_{2} \\
& \mathrm{BE}=4.03 \mathrm{eV} / \text { atom } \\
& \Delta E=0.92
\end{aligned}
$$
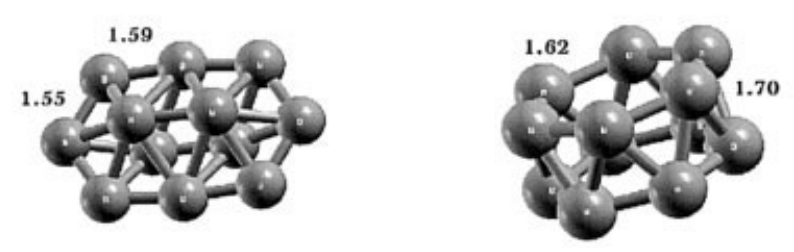

$$
\begin{aligned}
& \mathrm{B}_{12}-\mathrm{III}, \text { singlet } \\
& \mathrm{C}_{2 h} \\
& \mathrm{BE}=4.64 \mathrm{eV} / \text { atom } \\
& \Delta E=2.04
\end{aligned}
$$

$$
\begin{aligned}
& \mathrm{B}_{12}-\mathrm{IV}, \text { singlet } \\
& \mathrm{C}_{1} \\
& \mathrm{BE}=4.62 \mathrm{eV} / \text { atom } \\
& \Delta E=2.19
\end{aligned}
$$
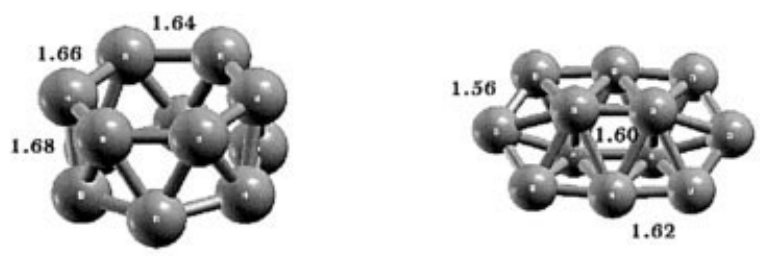

FIGURE 1. Isomeric configurations of $\mathrm{B}_{12}$ and $\mathrm{B}_{12}^{+}$: energy differences $(\Delta E$ in $\mathrm{eV})$ relative to the lowest-energy configuration, symmetry, spin multiplicity, and binding energy (eV/atom) obtained using the $6-31 \mathrm{G}(d, p)$ basis set.

energy configuration of $\mathrm{B}_{10}$ in $C_{2 h}$ symmetry $[18,19$, 23]. The double-ring isomer is the next low-energy structure (i.e., $\mathrm{B}_{12}-\mathrm{IV}$ ). Even though it is less stable relative to the convex $C_{1}$ structure of $B_{12}$, it is predicted to be extremely stable for large boron clusters [28]. The icosahedra unit, which is a fragment of a bulk $[7,8,16]$, is found to be more than $3 \mathrm{eV}$ higher in energy than the ground state of $B_{12}$.

The ionization-induced changes in ordering of the lowest-energy isomers of $\mathrm{B}_{12}$ are found to be small. The ground state of $B_{12}^{+}$remains to be the convex $C_{1}$ structure, as also predicted by a previous study [12]. The next isomer is a $C_{2}$ planar structure at $0.92 \mathrm{eV}$ above the $C_{1}$ isomer. The ordering of the next two lowest-energy isomers of $\mathrm{B}_{12}^{+}$clusters are almost same as that in the neutral clusters. In this case, double-ring with $C_{1}$ symmetry and cage-type $C_{2 h}$ structure are nearly degenerate with energy difference $(\Delta E)$ of $0.03 \mathrm{eV}$. The optimized geometry of the $\mathrm{B}_{12}^{+}$icosahedra is slightly distorted from its $I_{h}$ symmetry and is found to be $2.78 \mathrm{eV}$ higher in energy relative to the ground state. The ionization introduces small changes in the bond lengths and bond angles of the ionized clusters as compared to their corresponding neutral species.

The stability of the isomeric configurations of $B_{12}$ can be assessed in terms of the binding energy per atom (BE) as: $E_{b}\left[\mathrm{~B}_{n}\right]=-\left(E\left[\mathrm{~B}_{n}\right]-n E(\mathrm{~B})\right) / n$, where $E$ is the total energy of the system and $n$ is the total number of atoms. For the lowest-energy structure of $\mathrm{B}_{12}, \mathrm{BE}$ is $4.81 \mathrm{eV} /$ atom. Our calculations shows that $\mathrm{BE}$ increases from $\mathrm{B}_{6}{ }^{*}$ (3.95 eV/atom) to $\mathrm{B}_{12}$. This is consistent with the increasing trend of $\mathrm{BE}$ with the cluster-size approaching to the cohesive energy of about $6.0 \mathrm{eV}$ of the bulk boron [29]. The calculated binding energy per atom for the ground state of $\mathrm{B}_{12}^{+}$is $4.11 \mathrm{eV} /$ atom.

The vertical ionization potential is defined as the energy difference between the cationic and neutral clusters with both at the optimized geometry of the neutral cluster (i.e., $\mathrm{IP}_{\text {vertical }}=E_{q=0}-E_{q=+1}$, where $E$ is the total energy of the cluster and $q$ is the

*Details of the results on $\mathrm{B}_{6}$ can be obtained from the authors. 
charge on the cluster). The adiabatic ionization potential is defined as the energy difference between the cationic and neutral clusters at their own respective optimized geometries. The calculated vertical and adiabatic IP values are 8.52 and $8.40 \mathrm{eV}$, respectively. The experimental IP value [10] is reported to be $8.20 \mathrm{eV}$. The high value of IP for $\mathrm{B}_{12}$ is mainly due to the closed-shell electronic configuration in its ground state that provides a high stability of the cluster configuration.

\section{CHEMICAL BONDING AND ELECTRONIC PROPERTIES}

The chemical bonding of boron is known to be dominated by its electron-deficient character [21]. Thus, multi-center bonds such as the three-center triangular $\mathrm{B}-\mathrm{B}-\mathrm{B}$ unit become a key bonding feature in boron compounds to accommodate its electron deficiency [23]. It then results in the tendency of the boron atoms to prefer configuration based on a polyhedral unit (e.g., $B_{12}$ icosahedra unit) in which the triangular faces prevail. However, at the cluster level, calculations predict the preference of planar isomers over 3D isomers, including the icosahedra unit. To understand the difference in the preferred configurations at the cluster and bulk level, we have performed analysis of molecular orbitals for planar, double-ring, and icosahedra isomers of $B_{12}$.

The natural bond orbital (NBO) analysis [30] finds that the isomeric configurations considered here prefer $\mathrm{sp}^{2}$ hybridization, as the case with $\mathrm{B}_{6}$. For the lowest-energy configuration (i.e., $\mathrm{B}_{12}-\mathrm{I}$ ), the natural electron configuration for the atoms are (core) $2 \mathrm{~s}^{0.88} 2 \mathrm{p}^{2.03}$, (core) $2 \mathrm{~s}^{0.82} 2 \mathrm{p}^{2.09}$, and (core) $2 \mathrm{~s}^{0.55} 2 \mathrm{p}^{2.66}$, whereas for the $3 \mathrm{D}$ double-ring structure (i.e., $\mathrm{B}_{12}-\mathrm{IV}$ ), the natural electron configuration for the atoms is (core) $2 s^{0.76} 2 p^{2.22}$. On the other hand, the natural electron configurations of the the icosahedra isomer are (core) $2 \mathrm{~s}^{0.88} 2 \mathrm{p}^{2.12}$, (core) $2 \mathrm{~s}^{0.66} 2 \mathrm{p}^{2.31}$, and (core) $2 \mathrm{~s}^{0.67} 2 \mathrm{p}^{2.30}$. Also, Mulliken-partitioned atomic charge analysis suggests a negligible charge transfer among atoms in the $\mathrm{B}_{12}$ isomers. Thus, the mixing of $2 s$ and $2 p$ orbitals among boron atoms in the clusters is expected to play an important role in forming the covalent bond that stabilizes the configurations shown in Figure 1. A comparison of some of the molecular orbitals (MOs) of the convex planar structure and double-ring configuration of the neutral $B_{12}$ is shown in Figure 2. A similar analysis was also performed for $\mathrm{B}_{12}^{+}$isomers, which is not shown

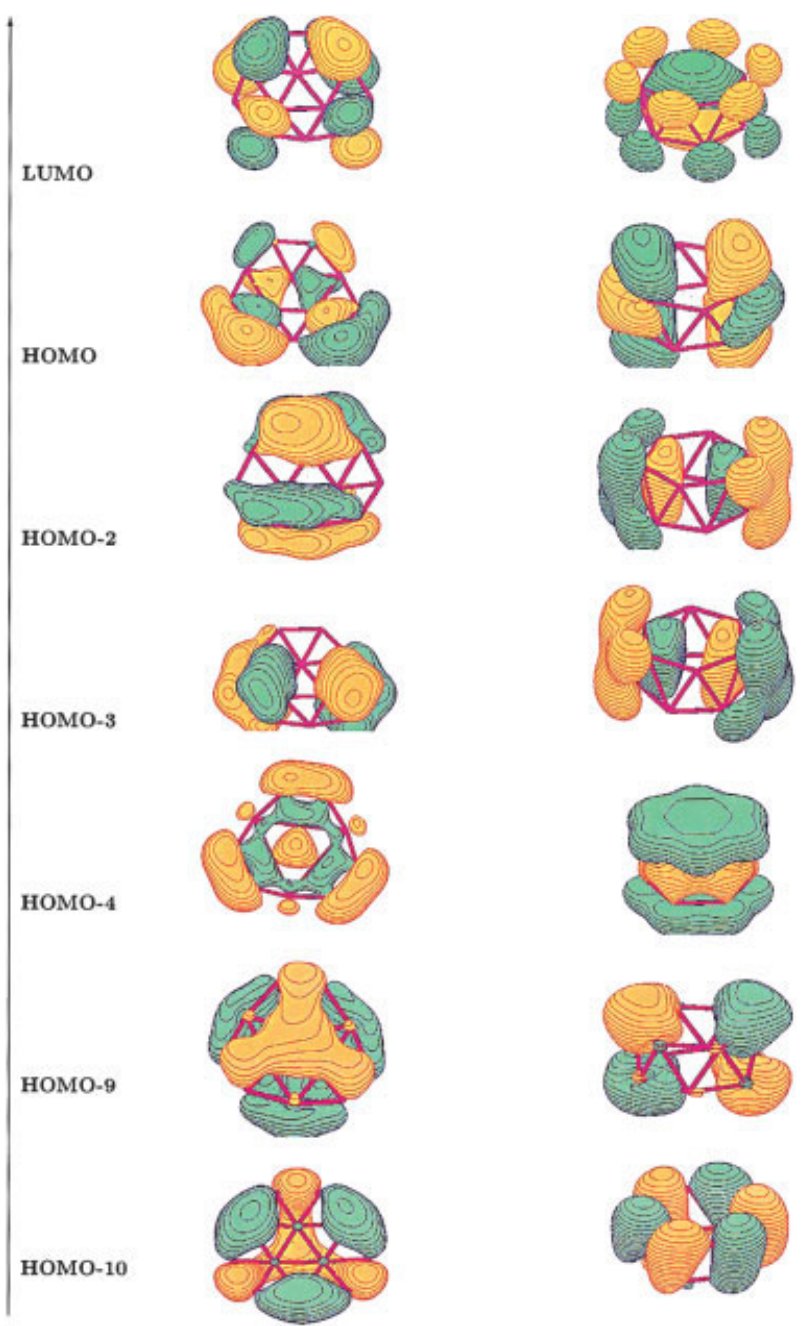

FIGURE 2. Some of the molecular orbitals (MOs) of convex planar and double-ring configurations of $\mathrm{B}_{12}$. [Color figure can be viewed in the online issue, which is available at www.interscience.wiley.com.]

here. In Figure 2, we use the notation HOMO- $n$ to represent the (30-n)th occupied molecular orbital in $B_{12}$, because there are 30 occupied molecular orbitals associated with 12 boron atoms in each cluster.

In the convex planar structure (i.e., $\mathrm{B}_{12}-\mathrm{I}$ ), the lowest unoccupied molecular orbital (LUMO) shows a localized $\pi$ bonding, while both highest occupied molecular orbital (HOMO) and HOMO-1 orbital show double-degenerate localized $\sigma$ bonds. The double degeneracy of the $\sigma$ bond leads to a delocalization of the cluster charge density. Besides, the double degeneracy of HOMO-2 and HOMO-3 orbitals, which is effectively due to the delocalized $\pi$ bonds, is found to be benzene-like in nature [23]. The multicentered, delocalized $\sigma$ bonds are present 
in HOMO-4 and HOMO-10 orbitals. It is noteworthy that the fully delocalized $\pi$ bonding present in the HOMO-9 orbital shows that the aromaticity that was found in benzene is also present in this neutral boron cluster.

The double-ring isomer (i.e., $\mathrm{B}_{12}$-IV) with a diameter of $3.2 \AA$ is composed of two rings of 6 atoms each, $1.62 \AA$ apart, arranged in a staggered configuration. Each ring is rotated by an angle of $\pi / 6$ with respect to the other ring in order to form the staggered configuration. Such a staggered doublering formation facilitates the $\mathrm{sp}^{2}$ hybridization, thereby making it comparatively stable among 3D structures. The charge density of $\mathrm{HOMO}$ and LUMO given in Figure 2 shows a strongly localized $\pi$ and $\sigma$ bond, respectively. The HOMO shows a $\pi$ bond between the atoms of each ring, while the LUMO shows a lateral p-p overlap between the atoms of both rings. The HOMO and (HOMO- 1 ) orbitals are doubly degenerate, giving rise to an effective delocalization. The double degeneracy of HOMO-2 and HOMO- 3 orbitals shows a localized $\sigma$ bonding, as compared to the delocalized $\pi$ bonding observed in the convex planar isomer. The delocalized $\pi$ bonding is also seen in HOMO-4 and HOMO-9 orbitals, and the HOMO-10 orbital shows the localized $\sigma$ bonding.

Thus, a comparison of the molecular orbitals of the convex planar and double-ring isomers suggests that the order of $\pi$ and $\sigma$ orbitals is reversed for HOMO and LUMO, respectively. This fact, together with a relatively larger degree of delocalized orbitals in the planar configuration, appears to be the dominant cause for the preference of the planar configuration in the ground state of neutral and cationic $B_{12}$ clusters. We also note here that the high strain energy due to a smaller diameter of the ring may contribute in making the double-ring isomer less competitive in becoming the lowest-energy configuration of $B_{12}$.

Besides chemical bonding, the coordination number of boron atoms is expected to play an important role in determining the cluster stability. In bulk, the coordination number of boron atoms is 5 [31]. On the other hand, the calculated (average) coordination number of boron atoms in planar and double-ring configurations is 4 as compared to that of 5 in the icosahedra configuration. However, the values of $\mathrm{BD}$ (i.e., 2-center bond based on Lewis structure) calculated from the NBO analysis [29] are 9 and 1 for planar and icosahedra isomers, respectively. This distinct difference in the values of BD suggests that the icosahedra configuration (which is a fragment of a bulk) is not energetically preferred despite of having a high coordination number for boron atoms.

It is well known that stability of a given cluster is reflected in the HOMO-LUMO gap. The HOMOLUMO gap for neutral and cationic lowest-energy structure of $\mathrm{B}_{12}$ are 3.61 and $1.51 \mathrm{eV}$. In general, ionized $\mathrm{B}_{12}$ clusters have a smaller gap relative to the corresponding neutral isomers. Given that the larger HOMO-LUMO gap generally refers to a higher value of the chemical hardness [32], ionized boron clusters appear to be chemically more reactive than the corresponding neutral clusters.

\section{VIBRATIONAL FREQUENCIES}

The neutral and cation isomers of $\mathrm{B}_{12}$ considered in this work are at local minima on the potential energy surface and their corresponding 3N-6 vibrational modes are shown in Figure 3, which excludes the translational and rotational modes frequencies. We are not aware of any previous theoretical study on the vibrational properties of $B_{12}$ and $B_{12}^{+}$. It is to be noted here that $B_{12}^{+}$is reported to be in transition state at the level of local spin density theory [11].

The calculated vibrational frequencies of $\mathrm{B}_{12}$ lie in the range of $200-1328 \mathrm{~cm}^{-1}$. The distribution of the vibrational frequencies of both neutral and cation isomers shows the similar trend. The frequencies in the cation isomers are generally lower than the corresponding neutral isomers. The lowest frequencies in the ground state of neutral and cationic isomers are $200.2 \mathrm{~cm}^{-1}$ and $107.7 \mathrm{~cm}^{-1}$, respectively. The highest frequencies in the ground state of neutral and cationic isomers are $1327.5 \mathrm{~cm}^{-1}$ and $1304.7 \mathrm{~cm}^{-1}$, respectively. The lower-frequency region of both neutral and cationic $B_{12}$ is associated with the bending modes, while the higher-frequency region $\left(>1000 \mathrm{~cm}^{-1}\right)$ is dominated by the stretching modes. The highest-frequency mode of the ground state of $\mathrm{B}_{12}$ and $\mathrm{B}_{12}^{+}$is associated with an asymmetric stretching of boron atoms located at the cluster surface.

From computational standpoint of view, the prediction of IR intensities can be accomplished by using the mixed second derivatives of energy with respect to geometric motion and an external electric field by permitting estimation of changes in the dipole moment as a function of vibrations. The intensity $(\mathrm{km} / \mathrm{mol})$ of an IR absorption band is proportional to the square of change in molecular electric dipole moment $\mu$ in normal coordinates (i.e., $I_{i}=$ const $\left.\times\left(\partial \mu / \partial Q_{i}\right)^{2}\right)$ [33]. 


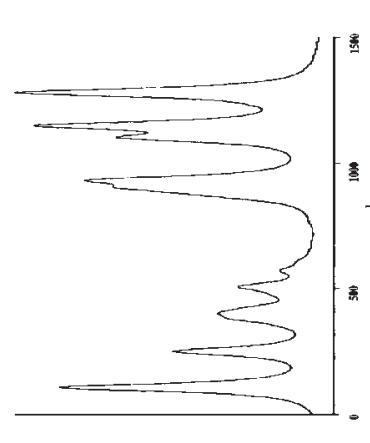

IR Intensity/(KM/Mole)

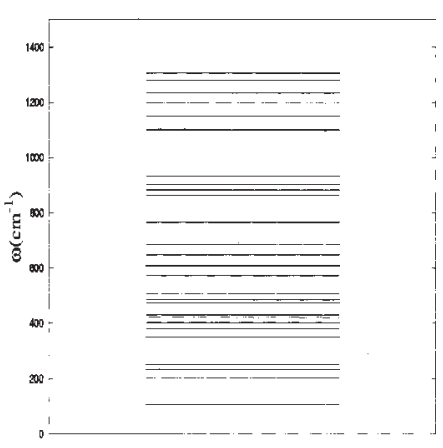

$\mathrm{B}_{12}^{+} \mathbf{I}$ convex (doublet)

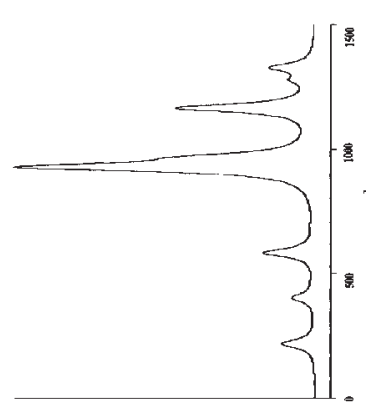

IR Intensity/(KM/Mole)

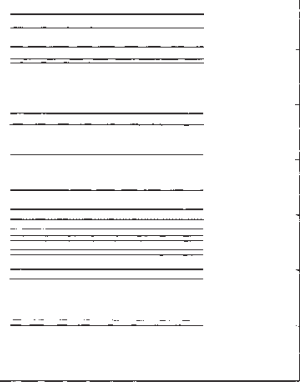

$\mathbf{B}_{12}$-I convex (singlet)

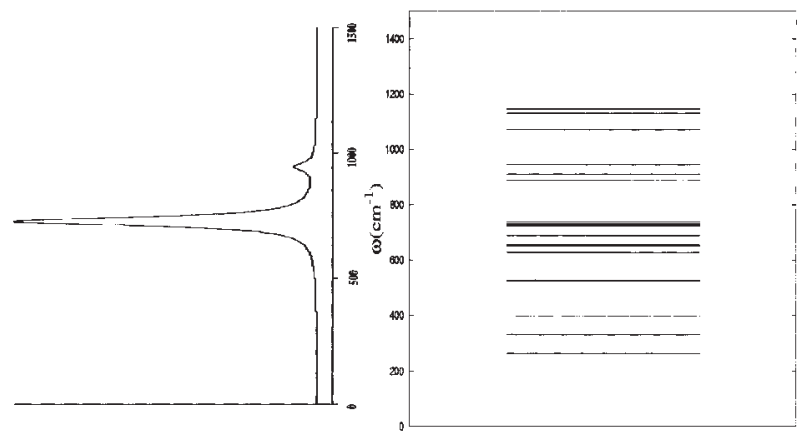

IR Intensity/(KM/Mole)

$\mathbf{B}_{12}$-IV double-ring (singlet)
FIGURE 3. The vibrational frequencies and the corresponding IR spectra of the $\mathrm{B}_{12}$ and $\mathrm{B}_{12}^{+}$clusters.

The IR spectra of the lowest-energy configuration of neutral (i.e., $\mathrm{B}_{12}-\mathrm{I}$ ), cation (i.e., $\mathrm{B}_{12}^{+}-\mathrm{I}$ ), and neutral double-ring (i.e., $\mathrm{B}_{12}-\mathrm{IV}$ ) is shown in Figure 3 . The neutral lowest-energy configuration has its IR active modes located in the region around 928 $\mathrm{cm}^{-1}$ and $1165 \mathrm{~cm}^{-1}$, which are expected to be in $\mathrm{A}_{1}$ and $\mathrm{E}$ mode if the convex planar structure is to be in $C_{3 v}$ symmetry [34]. The cationic ground-state structure has multiple IR active modes in the regions $108,251,933,1149$, and $1280 \mathrm{~cm}^{-1}$. The maximum peak is located at $1280 \mathrm{~cm}^{-1}$, which is associated with asymmetric stretching of three apex boron atoms. The IR active regions in both neutral and cationic ground state are quite similar with each other in the high-frequency region. Due to dissimilar atomic charges, the cationic isomer has another IR active mode region in the lower-frequency range. On the other hand, the neutral double-ring structure has only one unique IR active mode in its IR spectra at about $730 \mathrm{~cm}^{-1}$, and is mainly associated with three stretching modes that lie closely to each other at $723 \mathrm{~cm}^{-1}, 724 \mathrm{~cm}^{-1}$, and $730 \mathrm{~cm}^{-1}$. These three IR active modes are expected to be in $\mathrm{A}_{1}, \mathrm{~B}_{1}$, and $\mathrm{B}_{2}$ mode if the double-ring is to be in $C_{2 v}$ symmetry [34]. At $I_{\max }$ of about $730 \mathrm{~cm}^{-1}$, diameter of both the rings are found to expand and contract in an opposite way that acts like a "breathing mode" due to its unique geometrical structure.

\section{STATIC DIPOLE POLARIZABILITY}

Measurements of static dipole polarizability are frequently used by experimentalists to characterize the nature of atomic, molecular, and cluster species. Physically, it describes the response of the electronic cloud of the given cluster in the presence of an external static electric field. Instead of giving an exhaustive description of different characters in each isomers, we are presenting some of the features predicted by calculations in this study. In general, the calculated dipole moments of the neutral and cationic $\mathrm{B}_{12}$ show a close relationship with the structural symmetry of the cluster. The convex planar structure (i.e., $\mathrm{B}_{12}-\mathrm{I}$ ), which does not have a center of symmetry, has nonzero dipole moments, whereas cage-type (i.e., $\mathrm{B}_{12}$-III) and double-ring (i.e., $B_{12}$-IV) structures have zero dipole moments.

A direct comparison with the parallel $\left(\alpha_{x x}\right)$, transverse $\left(\alpha_{y y}\right)$, and perpendicular $\left(\alpha_{z z}\right)$ components of the static dipole polarizability of different isomers in the $\mathrm{B}_{n}$ series reported in the previous studies [35-37] is not possible because of the different orientations of $x, y$ and $z$ axes chosen in calculations. Thus, we have calculated the directional averaged static polarizability, $\alpha_{\text {avg }}=\left(\alpha_{x x}+\alpha_{y y}+\right.$ $\left.\alpha_{z z}\right) / 3$, to extract a general trend with the increase in the cluster size. Table I shows the calculated dipole moments and the static polarizability tensor along the $x, y$, and $z$ directions with the total dipole moment and directional averaged static polarizability of the low-energy configurations of $\mathrm{B}_{12}$ and $\mathrm{B}_{12}^{+}$.

The directional averaged static polarizability per atom (i.e., $\alpha_{\text {avg }} / n$, where $n$ is the total number of boron atoms in the cluster) shows overall decrease from 17.63 a.u. to 12.23 a.u. in going from $B_{3}^{37}$ to $B_{12}$. From Table I, we observe that the ionization of the 
TABLE I

Calculated dipole moment ( $\mu_{i}$ in debye), components of the static polarizability tensor $\left(\alpha_{j j}\right.$ in a.u.), and directional averaged static polarizability ( $\alpha_{\text {avg }}$ in a.u.) for low-energy configurations of $B_{12}$ and $B_{12}^{+}$.

\begin{tabular}{|c|c|c|c|c|c|c|c|c|}
\hline \multirow[b]{2}{*}{ Property } & \multicolumn{4}{|c|}{$\mathrm{B}_{12}, Q=0$} & \multicolumn{4}{|c|}{$\mathrm{B}_{12}, \mathrm{Q}=+1$} \\
\hline & $\mathrm{B}_{12}-\mathrm{I}$ & $\mathrm{B}_{12}-\mathrm{II}$ & $\mathrm{B}_{12}-\mathrm{III}$ & $\mathrm{B}_{12}-\mathrm{IV}$ & $\mathrm{B}_{12}^{+}-\mathrm{I}$ & $\mathrm{B}_{12}^{+}-\mathrm{II}$ & $\mathrm{B}_{12}^{+}-\mathrm{III}$ & $\mathrm{B}_{12}^{+}-\mathrm{IV}$ \\
\hline$\mu_{x}$ & -0.0004 & 0.0000 & 0.0000 & 0.0002 & 1.0654 & 0.0000 & 0.0000 & -0.0019 \\
\hline$\mu_{y}$ & 0.0017 & 0.0000 & 0.0000 & -0.0001 & 0.1008 & 0.0000 & 0.0000 & 0.0002 \\
\hline$\mu_{z}$ & 2.0752 & 0.0011 & 0.0000 & 0.0000 & 1.2516 & -0.0017 & 0.0000 & -0.0039 \\
\hline$\mu_{\text {Tot }}$ & 2.0752 & 0.0011 & 0.0000 & 0.0002 & 1.6467 & 0.0017 & 0.0000 & 0.0044 \\
\hline$\alpha_{x x}$ & 186.24 & 172.45 & 91.47 & 135.25 & 187.43 & 173.65 & 84.55 & 129.86 \\
\hline$\alpha_{y y}$ & 185.76 & 236.95 & 183.63 & 135.03 & 206.20 & 230.76 & 168.64 & 126.15 \\
\hline$\alpha_{z z}$ & 68.36 & 69.70 & 129.43 & 110.35 & 65.92 & 60.91 & 126.34 & 104.45 \\
\hline$\alpha_{\text {avg }}$ & 146.79 & 159.70 & 134.84 & 126.88 & 153.18 & 155.11 & 126.51 & 120.15 \\
\hline
\end{tabular}

neutral cluster decreases the polarizability. The ground state of the neutral cluster (i.e., $\mathrm{B}_{12}-\mathrm{I}$ ) has a significantly less polarizability $\left(\alpha_{\text {avg }}=146.79\right.$ a.u. $)$ as compared to that of the chainlike structure $\left(\alpha_{\text {avg }}=241.60\right.$ a.u. $)$ reported in a previous study [37]. It is interesting to note that delocalization of the charge distribution in a given cluster configuration plays an important role in reducing the static dipole polarizability. The large difference in $\alpha_{\text {avg }}$ between the chainlike and convex planar structure may therefore be attributed to the localized $\pi$-electron population along chain direction [37] as compared to delocalized $\pi$ and $\sigma$ bonds in the convex planar configuration of $\mathrm{B}_{12}$.

\section{BASIS SETS: A COMPARISON OF SOME RESULTS OBTAINED USING 6-31G(d,p) AND 6-311G(df) BASIS SETS}

To test the reliability and accuracy of the $6-31 \mathrm{G}(d, p)$ basis set used in this study, we also performed DFT calculations for the first two low-energy geometries of $\mathrm{B}_{12}$ and $\mathrm{B}_{12}^{+}$, using the $6-311 \mathrm{G}(d f)$ basis set. Table II collects some of the results in terms of binding energy, $\Delta E, \omega_{\text {lowest }} \omega_{\text {highest }}$ and HOMO-LUMO gap of $\mathrm{B}_{12}$ and $\mathrm{B}_{12}^{+}$clusters showing no significant difference in either energetics or frequencies obtained using the $6-31 G(d, p)$ and 6-311G $(d f)$ basis sets. Furthermore, the calculated values of vertical IP and adiabatic IP using the $6-311 \mathrm{G}(\mathrm{df})$ basis set come out to be 8.81 and 8.68 $\mathrm{eV}$, respectively. The $6-31 \mathrm{G}(d, p)$ basis set yields values of 8.52 and $8.40 \mathrm{eV}$ for vertical IP and adiabatic IP, respectively.

\section{Conclusion}

The ground state of $\mathrm{B}_{12}$ and $\mathrm{B}_{12}^{+}$is predicted to be the convex planar $C_{1}$ configuration in contrast to the convex planar $C_{3 v}$ configuration reported by previous studies. The ionization-induced structural changes in $B_{12}$ are found to be very small. It is shown that the basic building block of the bulk crystal is energetically not preferred for the $B_{12}$ cluster despite of its high coordination number for the boron atoms. The $\mathrm{B}_{12}^{+}$isomers are found to be less stable than the corresponding neutral species. A very high IP value and a large HOMO-LUMO gap show the very high stability of the neutral cluster. A combination of delocalized charge density and multicentered bonds facilitates the planar structure to be more energetically preferable than other isomers in $\mathrm{B}_{12}$. The calculated vibrational frequencies lie in the range of $200-1328 \mathrm{~cm}^{-1}$. The IR spectra of both neutral and cationic $B_{12}$ show similar IR active modes in the higher-frequency region, but different modes in the lower-frequency region due to dissimilar atomic charges in the cationic $B_{12}$. A combination of charge delocalization and reduced dimensionality of structural configurations appears to play an important role in determining the static dipole polarizability of $\mathrm{B}_{12}$ clusters. These results can be used as a baseline for future research on electrooptical properties in nano devices based on boron. It is also observed that the use of the 6-311G (df ) basis set does not introduce a significant change in the structural energetics, electronic properties, and vibrational properties of $\mathrm{B}_{12}$ and $\mathrm{B}_{12}^{+}$. 
THEORETICAL STUDY OF VIBRATIONAL PROPERTIES OF NEUTRAL AND CATIONIC $\mathrm{B}_{12}$ CLUSTERS

TABLE II

Comparison of some results obtained using $6-31 G(d, p)$ and $6-311 G(d f)$ basis sets

for low-energy isomers of $B_{12}$ and $B_{12}^{+}$.

\begin{tabular}{|c|c|c|c|}
\hline Isomer & Property & $6-31 \mathrm{G}(d, p)$ basis set & $6-311 \mathrm{G}(d f)$ basis set \\
\hline \multirow[t]{6}{*}{$\mathrm{B}_{12}-\mathrm{I}, \mathrm{C}_{1}$} & $E$ & -297.971543 & -298.042294 \\
\hline & $\Delta E$ & 0.00 & 0.00 \\
\hline & $\mathrm{BE}$ & 4.81 & 4.75 \\
\hline & HOMO-LUMO gap & 3.61 & 3.60 \\
\hline & $\omega_{\text {lowest }}$ & 200.2 & 200.02 \\
\hline & $\omega_{\text {highest }}$ & 1327.5 & 1321.68 \\
\hline \multirow[t]{6}{*}{$\mathrm{B}_{12}-\mathrm{II}, \mathrm{C}_{2}$} & $E$ & -297.914032 & -297.989053 \\
\hline & $\Delta E$ & 1.56 & 1.45 \\
\hline & $\mathrm{BE}$ & 4.68 & 4.63 \\
\hline & HOMO-LUMO gap & 2.75 & 2.79 \\
\hline & $\omega_{\text {lowest }}$ & 62.9 & 58.83 \\
\hline & $\omega_{\text {highest }}$ & 1635.76 & 1634.16 \\
\hline \multirow{6}{*}{$\mathrm{B}_{12}^{+}-\mathrm{I}, \mathrm{C}_{1}$} & $E$ & -297.662832 & -297.723387 \\
\hline & $\Delta E$ & 0.00 & 0.00 \\
\hline & $\mathrm{BE}$ & 4.11 & 4.02 \\
\hline & HOMO-LUMO gap & 1.51 & 1.52 \\
\hline & $\omega_{\text {lowest }}$ & 107.7 & 102.24 \\
\hline & $\omega_{\text {highest }}$ & 1304.7 & 1300.7 \\
\hline \multirow[t]{6}{*}{$\mathrm{B}_{12}^{+}-\mathrm{II}, \mathrm{C}_{2}$} & $E$ & -297.629154 & -297.692217 \\
\hline & $\Delta E$ & 0.92 & 0.85 \\
\hline & $\mathrm{BE}$ & 4.03 & 3.95 \\
\hline & HOMO-LUMO gap & 1.95 & 1.94 \\
\hline & $\omega_{\text {lowest }}$ & 68.34 & 64.37 \\
\hline & $\omega_{\text {highest }}$ & 1609.76 & 1605.29 \\
\hline
\end{tabular}

Units: total energy, $E$ (hartree), energy difference relative to the ground state, $\Delta E$ (eV), binding energy, BE (eV/atom), HOMO-LUMO gap $(\mathrm{eV})$, lowest and highest vibrational frequency $\left(\omega_{\text {lowest }}, \omega_{\text {highest }}\right.$ in $\left.\mathrm{cm}^{-1}\right)$.

\section{ACKNOWLEDGMENT}

We gratefully acknowledge CSERC, MTU, for allowing us access to their computing facility. MDD gratefully acknowledges for providing the local hospitality at the Michigan Technological University.

\section{References}

1. Semiconductors-Basic Data, 2nd rev ed. Madelung, O., Ed.; Springer-Verlag: Berlin-Heidelberg-New York, 1996.

2. Lipscomb, W. L. Boron Hydrides; W. A. Benjamin: New York, 1963.

3. Muetterties, E. L. (Ed.) Boron Hydride Chemistry, Academic: New York, 1975.

4. Muetterties, E. L. (Ed.) The Chemistry Of Boron and Its Compounds, John Wiley: New York, 1967.

5. Jemmis, E. D.; Balakrishnarajan, M. M.; Pancharatna, P. D. Chem Rev 2002, 102, 93.
6. Jemmis, E. D.; Balakrishnarajan, M. M.; Pancharatna, P. D. J Am Chem Soc 2001, 123, 4313.

7. Perkins, C. L.; Trenary, M.; Tanaka, T. Phys Rev Lett 1996, 77,4772 .

8. Hubert, H.; Devouard, B.; Garvie, L. A. J.; O'Keeffe, M.; Buseck, P. R.; Petuskey, W. T.; McMillan, P. F. Nature 1998, 391, 376.

9. Hanley, L.; Anderson, S. L. J Phys Chem 1987, 91, 5161.

10. Hanley, L.; Whitten, J. L.; Anderson, S. L. J Phys Chem 1988, $92,5803$.

11. Boustani, I. Int J Quantum Chem 1994, 52, 1081.

12. Ricca, A.; Bauschlicher Jr, C. W. Chem Phys 1996, 208, 233.

13. Gu, F. L.; Yang, X.; Tang, A. C.; Jiao, H.; Scheleyer, P. V. R. J Comput Chem 1998, 19, 203.

14. Aihara, J. I. J Phys Chem A 2001, 105, 5486.

15. Fowler, J. E.; Ugalde, J. M. J Phys Chem A 2000, 104, 397.

16. Kawai, R.; Weare, J. H. J Chem Phys 1991, 95, 1151.

17. Ray, A. K.; Howard, I. A.; Kanal, K. M. Phys Rev B 1992, 45, 14247.

18. Boustani, I. Chem Phys Lett 1995, 240, 135.

19. Boustani, I. Phys Rev B 1997, 55, 16426. 


\section{LAU, DESHPANDE, AND PANDEY}

20. Niu, J.; Rao, B. K.; Jena, P. J Chem Phys 1997, 107, 132.

21. Alexandrova, A. N.; Boldyrev, A. I.; Zhai, H. J.; Wang, L. S.; Steiner, E.; Fowler, P. W. J Phys Chem A 2003, 107, 1359.

22. Ma, J.; Li, Z. H.; Fan, K. N.; Zhou, M. F. Chem Phys Lett 2003, $372,708$.

23. Zhai, H. J.; Kiran, B.; Li, J.; Wang, L. S. Nature Materials 2003 , 2,827 .

24. Alexandrova, A. N.; Boldyrev, A. I.; Zhai, H. J.; Wang, L. S. J Phys Chem A 2004, 108, 3509.

25. Costales, A.; Kandalam, A. K.; Pandey, R. J Phys Chem A 2003, 107, 4508.

26. Frisch, M.; Trucks, G. W.; Schlegel, H. B.; Scuseria, G. E.; Robb, M. A.; Cheeseman, J. R.; Zakrzewski, V. G.; Montgomery, J. A.; Stratmann, R. E.; Burant, J. C.; Dapprich, S.; Millam, J. M.; Daniels, A. D.; Kudin, K. N.; Strain, M. C.; Farkas, O.; Tomasi, J.; Barone, V.; Cossi, M.; Cammi, R.; Mennucci, B.; Pomelli, C.; Adamo, C.; Clifford, S.; Ochterski, J.; Petersson, G. A.; Ayala, P. Y.; Cui, Q.; Morokuma, K.; Malick, D. K.; Rabuck, A. D.; Raghavachari, K.; Foresman, J. B.; Cioslowski, J.; Ortiz, J. V.; Stefanov, B. B.; Liu, G.; Liashenko, A.; Piskorz, P.; Komaromi, I.; Gomperts, R.; Martin, R. L.; Fox, D. J.; Keith, T.; Al-Laham, M. A.; Peng, C. Y.; Nanayakkara, A.; Gonzalez, C.; Challacombe, M.; Gill, P. M. W.; Johnson, B. G.; Chen, W.; Wong, M. W.; Andres, J. L.; Head-Gordon, M.;
Replogle, E. S.; Pople, J. A. Gaussian 98. Gaussian, Inc.: Pittsburgh, PA, 1998.

27. Becke, A. D. J Chem Phys 1993, 98, 5468.

28. Chacko, S.; Kanhere, D. G.; Boustani, I. Phys Rev B 2003, 68, 035414.

29. Lide, D. R. CRC Handbook of Chemistry and Physics; CRC Press: Boca Raton, FL, 1995.

30. Carpenter, J. E.; Weinhold, F. J Mol Struct (Theochem) 1988, $169,41$.

31. Gersten, J. I.; Smith, F. I. The Physics and Chemistry of Materials; John Wiley: New York, 2001.

32. Pearson, R. G. Chemical Hardness; Wiley-VCH: WeinheimNew York, 1997.

33. Koch, W.; Holthausen, M. C. A Chemist's Guide to Density Functional Theory, 2nd ed.; Wiley-VCH: Weinheim-New York, 2002.

34. Cotton, F. A. Chemical Applications of Group Theory, 3rd ed.; John Wiley: New York, 1990.

35. Reis, H.; Papadopoulos, M. G. J Comp Chem 1999, 20, 679.

36. Reis, H.; Papadopoulos, M. G.; Boustani, I. Int J Quantum Chem 2000, 78, 131.

37. Abdurahman, A.; Shukla, A.; Seifert, G. Phys Rev B 2002, 66 , 155423. 